\title{
Absorption of Sound in Liquids by the Resonator-Decay Technique: A Critique
}

\author{
Martin Greenspan \\ Institute for Basic Standards, National Bureau of Standards, Washington, D.C. 20234
}

(March 13, 1972)

\begin{abstract}
(1) The resonator-decay and the reverberation methods for measurement of the absorption of sound in liquids are in wide use, but their theoretical foundation has not hitherto been investigated. The basic assumptions are in fact invalid, but under most practical conditions the errors are tolerable, at least for three common geometries. (2) The very large excess losses always observed at the lowerorder modes are identified with anelastic effects in the envelope, for the most part, and with viscous boundary-layer effects to a lesser, but very substantial extent. For modes of sufficiently high order these become negligible.
\end{abstract}

Key words: Acoustics; acoustic impedance; acoustic materials; acoustic parameters.

\section{Introduction}

There is little doubt that many of the best measurements of the absorption of sound in liquids, especially at the lower frequencies, have been made by "decay" or "reverberation" methods. In the first case, a resonator formed by the sample liquid and a suitable container is excited in a single mode; the attenuation coefficient, $\alpha$, is calculated from the decay rate after the excitation is cut off. The second case is much the same, except that the excitation is over a band of frequencies.

It is curious that it has always been tacitly assumed that $\alpha$ as measured on a resonator is the same as that for a plane running wave in free space. That this cannot be true in general is evident from an obvious counterexample-in the case of uniform expansion (wavelength large $r e$ dimensions) the losses are governed by the congressional viscosity, $\lambda+2 \mu / 3$, whereas in free space the losses are governed by $\lambda+2 \mu$.

In section 2 we analyze the decay for a single mode in general and apply the results to three common cases: radially symmetric vibrations of a sphere, first used by Leonard $[1946]^{1}$ and in wide use ever since; radially symmetric vibrations of a cylinder, apparently first suggested by Meyer and Tamm [Mudlers, 1948] and the general vibrations of a rectangular parallelepiped (box). It is found that for the geometries analyzed the assumption in question is in general false, although for suitable configurations

\footnotetext{
${ }^{1}$ Figures in brackets indicate the literature references at the end of this paper
}

the errors in measured values of $\alpha$ may well be small. But even if so, it seems that a method in such wide use, and upon which so much reliance is placed, should rest on a firmer foundation than it has hitherto.

Once precautions are taken to minimize radiation of sound from the resonator into the air and conduction of sound in the supports, the most important sources of error are probably dissipation in the envelope and dissipation in the boundary layer. Both Leonard [1950] and Wilson [1951] tried to calculate envelope losses in a sphere without much success. New calculations are presented in section 3. Boundary layer losses occur whenever the motion of the liquid near the wall has a component parallel to it. This was first pointed out to Leonard by R. J. Christensen, and it is on this account that radial modes of a sphere, for which losses are smaller than for any other configuration, were employed by Leonard and by so many others since. Elementary calculations of boundary layer losses, for which no great originality is claimed, are presented in section 4 for comparison purposes.

\section{Theory}

The rationale of the resonator decay method can be summarized as follows. In a plane running wave, the energy density is

$$
E=E_{0} e^{-2 \alpha x}
$$

in which $\alpha$ is the amplitude attenuation coefficient. The variation with time, for an observer moving with the wave at speed $c$, is

$$
E=E_{0} e^{-2 \alpha c t} .
$$


Logarithmic differentiation gives for the instantaneous fraction of $E$ lost per unit time

$$
\frac{1}{E} \frac{d E}{d t}=+2 \alpha c .
$$

This notion is now applied to a resonator. The total energy density is $T+W$, i.e., the sum of the kinetic and potential energy densities. The viscous energy loss per unit volume (heat conduction is here neglected for simplicity; for most liquids it is minor) is twice the dissipation function, $2 F$. The ratio $-2 F /(T+W)$ corresponds to the left side of eq (1), hence

$$
\alpha=\frac{1}{2 c} \frac{2 \int F d V}{\int(T+W) d V} \simeq \frac{1}{2 c} \frac{\int F d V}{\int W d V},
$$

the integrations being taken over the whole volume of liquid. The second expression on the right in eq (2) is not exact; its use will be justified shortly. The assumption we wish to test is that $\alpha$ in eq (2) is the same as that in eq (1).

In the foregoing, and in the sequel as well, all first-order quantities, such as pressure, are supposed to vary as cos $\omega t$. Second-order quantities, such as $T, W$, and $F$, will have terms which vary as $\cos ^{2} \omega t$, $\sin ^{2} \omega t$, or $\sin \omega t \cos \omega t$. We are interested in time averages of $T, W$, and $F$ only-integration over $t$ will yield coefficients of $\frac{1}{2}$ or zero, as the cause may be. We bear this in mind, suppress the factors which indicate time variation, and take all first-order quantities as root-mean-square.

The problem reduces to finding expressions for $T, W$, and $F$. These are gotten from the pressure and velocity fields, supposed known. Although these can be determined in principle (for simple shapes) the solutions are too cumbersome to be useful. Instead we make the approximation that the field is the resultant of two others, the first appropriate to an inviscid fluid (i.e., one having a potential) and the second, appreciable only in a relatively thin boundary layer, that of a locally plane shear wave launched at the boundary along the inward normal, the magnitudes of the two being adjusted so that the net tangential velocity on the liquid-solid interface is zero [Morse and Ingard, 1968]. The functions $T, W$, and $F$ are calculated from the potential-solution only.

The assumed velocity potential, $\psi$, satisfies the wave equation

$$
\nabla^{2} \psi=-k^{2} \psi=-\left(\omega^{2} / c^{2}\right) \psi
$$

The pressure is

$$
p=\rho \frac{d \psi}{d t}=i \omega \rho \psi
$$

in which $\rho$ is the undisturbed density, and the velocity is

$$
u_{i}=-\psi_{, i}(i=1,2,3) .
$$

The comma indicates differentiation. We need also the strain rate

$$
\dot{S}_{i j}=\frac{1}{2}\left(u_{i, j}+u_{i, j}\right)=-\psi, i j
$$

and the dilatation rate

$$
\dot{\Delta}=\dot{S}_{i i}=-\psi, i i=-\nabla^{2} \psi=k^{2} \psi .
$$

The summation convention is used throughout.

We are now in a position to calculate $T, W$, and $F$. First we note the constitutive equation

$$
T_{i j}=(-p+\lambda \dot{\Delta}) \delta_{i j}+2 \mu \dot{S}_{i j}
$$

in which $T_{i j}$ is the stress, $\lambda$ and $\mu$ the second and first viscosity coefficients respectively, and $\delta_{i j}$ the unit tensor. The strain energy density is given by

$$
2 W=T_{i j} S_{i j}=(-p+\lambda \dot{\Delta}) \delta_{i j} S_{i j}+2 \mu \dot{S}_{i j} S_{i j}
$$

In eq (9) $S_{i j}$ is in quadrature with $\dot{S}_{i j}$ and with $\dot{\Delta}$; the only surviving term is that in $p \delta_{i j} S_{i j}$ which equals $p \Delta$, and as $\Delta=\Delta / i \omega$, we have from eqs (3) and (4)

$$
2 W=\rho k^{2} \psi^{2} .
$$

The kinetic energy density is $\rho u_{i} u_{i} / 2=\rho \psi_{, i} \psi_{, i} / 2$, from eq (5). A special case of Green's theorem,

$$
\int_{V} \psi_{, i} \psi_{, i} d V+\int_{V} \psi \nabla^{2} \psi d V=-\int_{S} \psi \frac{d \psi}{d n} d S
$$

leads to the condition of resonance. It is easily seen that it is equivalent to

$$
\int_{V}(T-W) d V=-\frac{\rho}{2} \int_{S} \psi \frac{d \psi}{d n} d S
$$

At resonance, the total kinetic and potential energies are equal so that the right-hand side of eq (12) vanishes. A sufficient condition is that everywhere on the outer surface (of the container, which we treat as a fluid without too much error because it is relatively thin) either the pressure (proportional to $\psi$ ) or the normal velocity (proportional to $d \psi / d n$ ) is zero. In practice, the resonator is operated at frequencies much above resonance (breathing mode) of the empty envelope. Hence the energy in the envelope is mostly kinetic and therefore most of the potential energy is in the liquid. A good approximation to the total energy is therefore $2 \int_{v} W d V$, the integration being taken over the volume of the liquid. This explains the substitution of $2 W$ for $T+W$ in eq (2).

The dissipation function is given by

$$
2 F=T_{i j} \dot{S}_{i j}=(-p+\lambda \dot{\Delta}) \delta_{i j} \dot{S}_{i j}+2 \mu \dot{S}_{i j} \dot{S}_{i j}
$$

Here $p$ is in quadrature with $S_{i j}$ and eq (13) reduces to 


$$
\begin{aligned}
2 F= & (\lambda+2 \mu) \dot{\Delta}^{2}+4 \mu\left(\dot{S}_{12}^{2}+\dot{S}_{23}^{2}+\dot{S}_{31}^{2}-\dot{S}_{11} \dot{S}_{22}\right. \\
& \left.-\dot{S}_{22} \dot{S}_{33}-\dot{S}_{33} \dot{S}_{11}\right)=(\lambda+2 \mu) k^{4} \psi^{2}+4 \mu I_{2}
\end{aligned}
$$

in which $I_{2}$ is the quadratic strain-rate invariant. Equation (14) together with eqs (2) and (10) gives finally,

$$
\alpha=\frac{\int F d V}{2 c \int W d V}=\frac{(\lambda+2 \mu) \omega^{2}}{2 \rho c^{3}}+\frac{2 \mu \int I_{2} d V}{\rho c k^{2} \int \psi^{2} d V} .
$$

We recognize the first term on the right-hand side of eq (15) as the plane-wave value of $\alpha$; therefore the condition that the resonator-decay experiment yield the plane-wave value of $\alpha$ is that

$$
\int I_{2} d V=0 .
$$

For reference we repeat

$$
I_{2}=\dot{S}_{12}^{2}+\dot{S}_{23}^{2}+\dot{S}_{31}^{2}-\dot{S}_{11} \dot{S}_{22}-\dot{S}_{22} \dot{S}_{33}-\dot{S}_{33} \dot{S}_{11}
$$

We note two special cases. In the case of a plane wave, running or standing along $x_{1}$, only $\dot{S}_{11}$ in eq (17) is not zero, so $I_{2}=0$ and eq (15) gives the known result. In the case of uniform expansion, the shear rates in eq (17) are zero and $S_{11}=S_{22}=S_{33}=S$ (say), uniform in space. Then $\dot{\Delta}=3 \dot{S}$ and $I_{2}=-3 \dot{S}^{2}=-\frac{1}{3} \dot{\Delta}^{2}$. With this substitution, eq (15) becomes

$$
\alpha=\frac{(\lambda+2 \mu / 3) \omega^{2}}{2 \rho c^{3}},
$$

which is the correct result, $\lambda+2 \mu / 3$ being the compressional viscosity.

\subsection{Sphere, Radial Mode}

Here we use spherical coordinates, $r, \theta, \phi$, and suppose that the potential, $\psi$, depends on $r$ only. Then the velocities are

$$
u_{r}=-\frac{d \psi}{d r}, u_{\theta}=u_{\phi}=0
$$

The nonzero strain rates are

$$
\dot{S}_{r r}=-\frac{d^{2} \psi}{d r^{2}}, \dot{S}_{\theta \theta}=\dot{S}_{\phi \phi}=\frac{u_{r}}{r}=-\frac{1}{r} \frac{d \psi}{d r},
$$

so that, from eq (17)

$$
I_{2}=-\frac{2}{r^{2}} \frac{d \psi}{d r}\left(\frac{d \psi}{d r}+2 r \frac{d^{2} \psi}{d r^{2}}\right),
$$

and with $d V=4 \pi r^{2} d r$,

$$
\int I_{2} d V=-4 \pi\left[r\left(\frac{d \psi}{d r}\right)^{2}\right]_{0}^{a}=-4 \pi a u_{a}^{2}
$$

in which $a$ is the radius of the sphere and $u_{a}=\left(u_{r}\right)_{r=a}$.
Equations (18) and (15) together show that for this case the resonator-decay result underestimates $\alpha$ except only for $u_{a}=0$, corresponding to a rigid container.

According to eq (14) for $2 F$ the excess in $\alpha, \Delta \alpha$, can be estimated from

$$
\frac{\Delta \alpha}{\alpha}=\frac{4 \mu \int_{V} I_{2} d V}{(\lambda+2 \mu) k^{4} \int_{V} \psi^{2} d V} .
$$

For the radial mode of a sphere the velocity potential for unit amplitude is

$$
\psi=\frac{\sin k r}{r} .
$$

Equations (18), (19) and (20) yield

$$
\frac{\Delta \alpha}{\alpha}=-\frac{6}{k a} \frac{\alpha_{c 1}}{\alpha} \frac{\left(\cos k a-\frac{\sin k a}{k a}\right)^{2}}{k a-\sin k a \cos k a} .
$$

In eq (21) we have written $\alpha_{c 1} / \alpha$ for $4 \mu / 3(\lambda+2 \mu)$ where $\alpha_{c 1}$ corresponds to the "classical" relationship $3 \lambda+2 \mu=0$. To use eq (21) for a particular case we need the values of $k$ corresponding to the various resonances. Although these are readily calculated, we content ourselves with an examination of the worst case, i.e., the pressure-release case, $p_{a}=0$. Then in eq (2l) $\sin k a=0, k a=n \pi, \cos k a= \pm 1$. We have

$$
\frac{\Delta \alpha}{\alpha}=-6 \frac{\alpha_{c 1}}{\alpha} \frac{1}{n^{2} \pi^{2}} .
$$

In the case of water, for which $\alpha_{c 1} / \alpha$ is about $\frac{1}{3}$, the "error" is large for the very low-order modes; about 20 percent for $n=1$, five percent for $n=2$, etc., but is less than one percent for $n \geqslant 5$. For most other liquids $\Delta \alpha / \alpha$ is even smaller. As we shall see, there are other, more compelling, reasons to operate at the high-order modes, so that for practical purposes the spherical resonator, other things aside, has a reasonably firm theoretical basis.

As a check on eq (2l) we note that for $k=0$ (uniform expansion) eq (21) yields the known result. ${ }^{2}$

\subsection{Cylinder, Radial-Axial Mode}

This case is not so simple, and the explicit expression for $\psi$ is required. If the mid-point of the axis is the origin of cylindrical coordinates, $r, \theta, z$, the potential is, for amplitude unity,

$$
\psi=J_{0}\left(k_{r} r\right) \cos \left(k_{z} z+\epsilon\right),
$$

with the radial and axial wave numbers $k_{r}$ and $k_{z}$ subject to

$$
k_{r}^{2}+k_{z}^{2}=k^{2}=\omega^{2} / c^{2} .
$$

${ }^{2}$ The indeterminate form in eq (21) is easily evaluated by expansion of the sine and cosine functions about $k a=0$. 
The velocities are

$$
u_{r}=-\frac{\partial \psi}{\partial r}=k_{r} J_{1} c, u_{\theta}=0, u_{z}=-\frac{\partial \psi}{\partial z}=k_{z} J_{0} s .
$$

Here and in the sequel we use abbreviations like $J_{1}$ for $J_{1}\left(k_{r} r\right)$ and $c$ for $\cos \left(k_{z} z+\epsilon\right)$. The strain rates are

$$
\begin{aligned}
& \dot{S}_{r r}=\frac{\partial u_{r}}{\partial r}=k_{r}^{2}\left(J_{0}-\frac{J_{1}}{k_{r} r}\right) c, \\
& \dot{S}_{\theta \theta}=\frac{u_{r}}{r}=k_{r}^{2} \frac{J_{1}}{k_{r} r} c, \\
& \dot{S}_{z z}=\frac{\partial u_{z}}{\partial z}=k_{z}^{2} J_{0} s, \\
& \dot{S}_{r z}=\frac{1}{2}\left(\frac{\partial u_{z}}{\partial r}+\frac{\partial u_{r}}{\partial z}\right)=-k_{r} k_{z} J_{1} s, \\
& \dot{S}_{r \theta}=\dot{S}_{\theta z}=0 .
\end{aligned}
$$

These, together with $d V=2 \pi r d r d z$ and the limits 0 to $a$ for $r$ and $-L$ to $L$ for $z$, give after some reduction

$$
\begin{aligned}
-\frac{1}{\pi k_{r}} \int I_{2} d V= & A+B\left[\sin \left(k_{z} L+\epsilon\right) \cos \left(k_{z} L+\epsilon\right)\right. \\
& \left.-\sin \left(-k_{z} L+\epsilon\right) \cos \left(-k_{z} L+\epsilon\right)\right]
\end{aligned}
$$

where

$$
\begin{aligned}
A= & 2 L J_{1}\left(k_{r} a\right)\left[k_{z}^{2} a J_{0}\left(k_{r} a\right)\right. \\
& \left.+\frac{k_{r}}{2} J_{1}\left(k_{r} a\right)\right]
\end{aligned}
$$

and

$$
\begin{aligned}
B= & k_{z} a\left\{k_{r} a\left[J_{0}^{2}\left(k_{r} a\right)+J_{1}^{2}\left(k_{r} a\right)\right]\right. \\
& \left.-J_{0}\left(k_{r} a\right) J_{1}\left(k_{r} a\right)\right\}+\frac{k_{r}}{2 k_{z}} J_{1}^{2}\left(k_{r} a\right) .
\end{aligned}
$$

For the integral in eq (25) to be zero it is sufficient that $J_{1}\left(k_{r} a\right)=0$, that either $\sin \left(k_{z} L+\epsilon\right)$ or $\cos$ $\left(k_{z} L+\epsilon\right)$ be zero, and that either $\sin \left(-k_{z} L+\epsilon\right)$ or $\cos \left(-k_{z} L+\epsilon\right)$ be zero. This follows from the independence of $k_{r}$ and $k_{z}$. Restated, a sufficient condition that the experiment measure the free-space value of $\alpha$ is that the cylindrical wall be rigid and that each end be either rigid or pressure-release. (Another condition is $k_{r}=0$, corresponding to a plane wave.)

We can calculate the error, $\Delta \alpha / a$, in the same way as for the sphere. In the special case of pressurerelease ends, we get

$$
\frac{\Delta \alpha}{\alpha}=-6 \frac{\alpha_{c 1}}{\alpha} \frac{k_{r}}{k^{4} a^{2}} \frac{J_{1}\left(k_{r} a\right)\left[k_{z} a J_{0}\left(k_{r} a\right)+\frac{k_{r}}{2} J_{1}\left(k_{r} a\right)\right]}{J_{0}^{2}\left(k_{r} a\right)+J_{1}^{2}\left(k_{r} a\right)} .
$$

Again, the worst case is for a pressure-release cylin- drical surface $\left[J_{1}\left(k_{r} a\right)=0\right]$ for which eq (26) becomes

$$
\frac{\Delta \alpha}{\alpha}=-3 \frac{\alpha_{c 1}}{\alpha} \frac{\lambda_{0, m}^{2}}{\left[\lambda_{0, m}^{2}+\left(\frac{n \pi a}{2 L}\right)^{2}\right]^{2}},
$$

in which $\lambda_{0, m}$ is the $m$ th zero of $J_{0}(x)$. Thus high mode numbers $m$ or $n$ (or both) insure a small "error." The conclusion is the same as for the sphere-the method is valid in practical cases.

\subsection{Rectangular Tank}

In rectangular coordinates, $x_{1}$, the potential, for amplitude unity, is

$$
\psi=c_{1} c_{2} c_{3} .
$$

Again, we use abbreviations like $c_{1}=\cos \left(k_{1} x_{1}+\epsilon_{1}\right)$. In eq (28) the $k_{i}$ are subject to

We have

$$
k_{1}^{2}+k_{2}^{2}+k_{3}^{2}=k^{2}=(\omega / c)^{2} .
$$

$$
\begin{gathered}
u_{1}=-\frac{\partial \psi}{\partial x_{1}}=k_{1} s_{1} c_{2} c_{3}, u_{2}=-\frac{\partial \psi}{\partial x_{2}}=k_{2} c_{1} s_{2} c_{3}, \\
u_{3}=-\frac{\partial \psi}{\partial x_{3}}=k_{3} c_{1} c_{2} s_{3},
\end{gathered}
$$

and

$$
\begin{aligned}
& \dot{S}_{11}=\frac{\partial u_{1}}{\partial x_{1}}=k_{1}^{2} c_{1} c_{2} c_{3}, \text { etc. } \\
& \dot{S}_{12}=\frac{1}{2}\left(\frac{\partial u_{2}}{\partial x_{1}}+\frac{\partial u_{1}}{\partial x_{2}}\right)=k_{1} k_{2} s_{1} s_{2} c_{3}, \text { etc. }
\end{aligned}
$$

The invariant $I_{2}$ of eq (17) will have three terms. The first is

$$
-\frac{1}{2} k_{1}^{2} k_{2}^{2} c_{3}^{2}\left[\cos 2\left(k_{1} x_{1}+\epsilon_{1}\right)+\cos 2\left(k_{2} x_{2}+\epsilon_{2}\right)\right] ;
$$

the other two are got by cyclic permutation of the subscripts. Integration over the range $-L_{i}$ to $L_{i}$ for $x_{i}$ yields an expression for $\int I_{2} d V$ containing terms of the form

$\sin \left(k_{i} L_{i}+\epsilon_{i}\right) \cos \left(k_{i} L_{i}+\epsilon_{i}\right)$

$$
-\sin \left(-k_{i} L_{i}+\epsilon_{i}\right) \cos \left(-k_{i} L_{i}+\epsilon_{i}\right)
$$

together with cross-products of these taken two at a time. The coefficients of these are various products of $L_{i}$ and powers of $k_{i}$. An account of the independence of these coefficients a sufficient condition that

$$
\int_{V} I_{2}=0
$$

is that each face be either rigid (sine term $=0$ ) or pressure-release $($ cosine term $=0)$. There are, of 
course, other special conditions. For instance if any two $k_{i}$ are zero the integrand $I_{2}$ vanishes - we are back to a plane standing wave.

The actual "errors" for any particular case could be calculated with some labor, but it seems hardly worthwhile in consideration of what we have already learned in the other cases.

\section{Losses in the Envelope}

\subsection{Sphere, Radial Modes}

The loss in a spherical shell oscillating in the radial (breathing) mode is not difficult to calculate if a simple expression is obtained for the real component of the impedance of the shell. It is the fraction $1 / Q_{E}$ of the stiffness reactance (not the mass reactance; the losses are associated with strain rates, not with inertia). The stiffness react ance is $M_{E} \omega_{0}^{2} / \omega, M_{E}$ being the mass of the envelope and $\omega_{0}$ its resonance angular frequency when empty. Hence the dissipation rate is

$$
D_{E}=\frac{M_{E} \omega_{0}^{2}}{Q_{E} \omega} u_{a}^{2},
$$

in which $u_{a}$ is the radial velocity at $r=a$, the interface. The approximation is here made that the radial velocity is constant throughout the thickness of the envelope. This expression is of use only if the variation of $Q_{E}$ with $\omega$ is known. For many solid materials, including glass, the $Q$ at frequencies of interest here is independent of frequency (elastic hysteresis) [Mason, 1950] and we shall take it as such. We seek the ratio of $D_{E}$, the dissipation rate in the envelope, to $D_{L}$; that in the liquid. For the latter we use, without significant error only the part $(\lambda+2 \mu) k^{4} \int_{V} \psi^{2} d V$ [eq (14)]. This and the value of $u_{a}$ in eq (29) are gotten from the potential in eq (20). The result is

$\frac{D_{E}}{D_{L}}=\frac{2 \rho_{E} c_{L} t \sin ^{2} k a}{(\lambda+2 \mu) Q_{E}(k a)^{2}}\left(\frac{\omega_{0}}{\omega}\right)^{2} \frac{(1-k a \cot k a)^{2}}{k a-\sin k a \cos k a}$,

in which $\rho_{E}$ and $t$ are the density and thickness of the envelope and $c_{L}$ is the speed of sound in the liquid. Equation (30) is meaningful in our context only for those values of $\omega($ and $k=\omega / c)$ which correspond to actual resonances.

One expression of the condition of resonance is that on the boundary between the liquid and the envelope the point admittance of the liquid equals that of the envelope. The former is

$$
\frac{u_{a}}{\rho_{a}}=\frac{-\partial \psi / \partial a}{i \omega \rho_{L} \psi_{a}}=\frac{1-k a \cot k a}{i \omega \rho_{L} a},
$$

$\rho_{L}$ being the density of the liquid, and the latter is

$$
y_{E}=1 / i \omega \rho_{E} t\left[1-\left(\frac{\omega_{0}}{\omega}\right)^{2}\right] \text {. }
$$

Equating these gives as the condition of resonance

$$
1-k a \cot k a=\frac{\rho_{L} a}{\rho_{E} t\left[1-\left(\frac{\omega_{0}}{\omega}\right)^{2}\right]} .
$$

This together with eq (30) gives, after much reduction

$$
\frac{D_{E}}{D_{L}}=\frac{1}{c_{L} Q_{E} \omega\left(\alpha / \omega^{2}\right)}\left(\frac{\omega_{0}}{\omega}\right)^{2}
$$

$$
\frac{R}{R^{2}-R\left[1-\left(\omega_{0} / \omega\right)^{2}\right]+(k a)^{2}\left[1-\left(\omega_{0} / \omega\right)^{2}\right]^{2}},
$$

in which we have written $R$ for $\rho_{L} a / \rho_{E} t$, and have eliminated $\lambda+2 \mu$ in favor of $\alpha / \omega^{2}$ from eq (15). We shall see that in most practical cases $\omega_{0} \ll \omega$ so that the bracketed expressions in eq (33) are nearly unity. Equation (33) is still not free of $\omega$ (or $k=\omega / c)$, but the righthand side is seen to be a monotonic function of $\omega$, so that the equation is usable as it stands; it is not necessary to solve the frequency equation, eq (31) explicitly.

It seems clear that, everything else being equal, there is no optimum thickness, $t$, of the envelope wall, because the wall loss would vanish at both extremesno wall and rigid wall. This notation is verified by eq (33) according to which $D_{E} / D_{L}$ has a maximum for $R=k a\left[1-\left(\omega_{0} / \omega\right)^{2}\right]$. $(R$ varies as $1 / t)$. The variation of the error, $D_{E} / D_{L}$ with frequency is very rapid. For a given set-up, and for liquids for which $\alpha / \omega^{2}$ is independent of frequency, eq (33) predicts that for low-order modes, such that $R \gg k a, D_{E} / D_{L}$ varies as $1 / \omega^{3}$, and for high-order modes, such that $R \ll k a$, it varies as $1 / \omega^{5}$. Thus we might expect, for a given experimental arrangement, very large errors at low frequencies and negligible errors for sufficiently high frequencies.

These conclusions can be checked against the results obtained by Wilson [1951] whose report contains all of the necessary details. The circles in figure 1 show $\alpha_{\text {obs }} / \alpha$, the ratio of the observed to the known attenuation in water ${ }^{3}$ at $4{ }^{\circ} \mathrm{C}$, as measured in a 12 liter borosilicate boiling flask. The errors at the low-order modes are seen to be enormous; the observed attenuation is too high by a factor of more than 100 at $16 \mathrm{kHz}$ (mode 3 ). Even at the ninth mode, about 46 $\mathrm{kHz}$, the factor is about 4. At the higher-order modes the factor remains constant at about 1.4.

Wilson's report [1951] gives all the data needed to calculate the error from eq (33). There is some question about the $Q$ of borosilicate however. The value most often cited, 1200, is taken from Mason [1950]. Wilson [1951] measured it as 2200 for longitudinal vibrations. Measurements made in our laboratory on two spherical flasks vibrating in a vacuum in the breathing mode give about 2700 at $4^{\circ} \mathrm{C}$.

The full lines in figure 1 show $1+\left(D_{E} / D_{L}\right)$ from eq (33) for $Q_{E}$ 's of 1000 and 3000. It is clear that eq (33) explains the low-frequency errors fairly well at least

\footnotetext{
${ }^{3}$ The "known values" are those extrapolated from high-frequency measurements on the supposition that $\alpha / \omega^{2}$ is independent of frequency.
} 


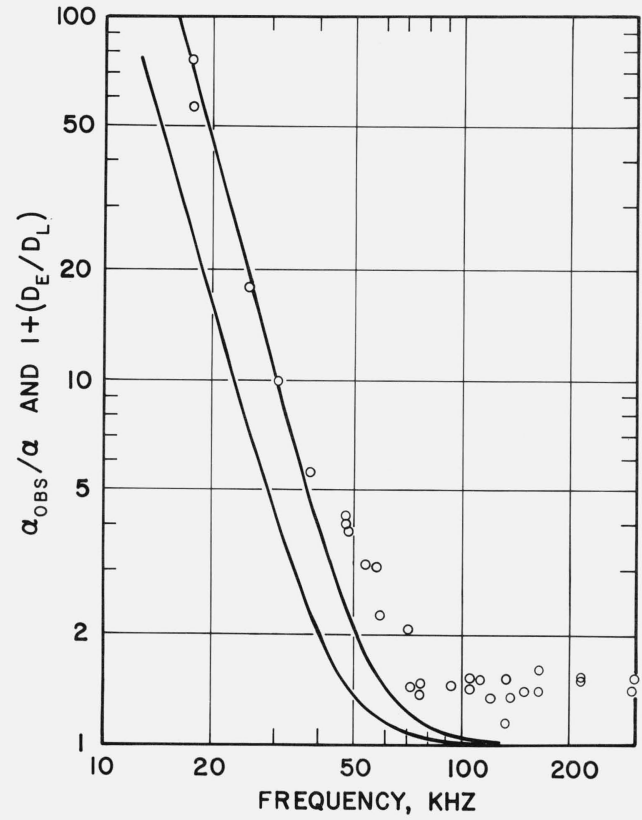

FigURE 1. Losses in water at $4{ }^{\circ} \mathrm{C}, 12$-liter spherical Pyrex flask. The circles show the ratios of the measured to the known values of the attenuation according to Wilson [1951]. The curves show $1+\left(D_{E} / D_{L}\right)$ from eq $(33)$; the upper curve is for $Q_{E}=1000$ and the lower for $Q_{E}=3000$

in a qualitative way. It must be remembered that there are other sources of error. Some of these will be discussed later. The constant error at high frequencies, which remains unexplained, is discussed in section 5 .

\subsection{Cylinder, Radial Axial Modes}

The calculation is carried out much as for the sphere. In the case of pressure-release ends, the result is

$\frac{D_{E}}{D_{L}}=$

$$
\frac{1}{c_{L} Q_{E} \omega\left(\alpha / \omega^{2}\right)}\left(\frac{\omega_{0}}{\omega}\right)^{2}\left(\frac{k_{r}}{k}\right)^{2} \frac{R}{R^{2}+\left(k_{r} a\right)^{2}\left[1-\left(\omega_{0} / \omega\right)^{2}\right]^{2}} .
$$

In deriving eq (34) the point admittance of the envelope was calculated in a manner similar to that for a sphere [eq (31)]. This is probably good enough for the $(l, 0,1)$ modes, but dubious for the $(l, 0, n>1)$ modes. A more ambitious attack was made by Lambert [1953], but his results are not applicable here. The point admittance of the liquid, at the interface, is

$$
\frac{u_{a}}{p_{a}}=\frac{-\partial \psi / \partial a}{i \omega \rho_{L} \psi_{a}}=\frac{k_{r} J_{1}\left(k_{r} a\right)}{i \omega \rho_{L} J_{0}\left(k_{r} a\right)},
$$

so that the condition of resonance is

$$
\frac{k_{r} a J_{1}\left(k_{r} a\right)}{J_{0}\left(k_{r} a\right)}=\frac{R}{1-\left(\omega_{0} / \omega\right)^{2}}
$$

in which again, $R=\rho_{L} a / \rho_{E} t$.
The main point of eq (34) is that the envelope losses for a cylinder behave like those for a sphere, that is, they can be very large or very small for a given setup, depending on whether the mode numbers are very low or very high.

\section{Losses in the Viscous Boundary Layer}

The viscous boundary-layer losses are calculated on the assumption that the boundary layer thickness, $d_{v}=\left(2 \mu / \rho_{L} \omega\right)^{1 / 2}$, is small relative to the interior dimensions of the resonator. The method is very old; a good simple account is given by Lambert [1953]. The viscous loss per unit area at the interface is given by

$$
\frac{d D_{B}}{d S}=\sqrt{\omega \rho_{L} \mu / 2} u_{t}^{2},
$$

in which $u_{t}$ is the tangential component of the fluid velocity at the interface as calculated from the potential.

It follows from eq (36) that if $u_{t}=0$ everywhere at the interface, the boundary-layer losses are zero. This is the case for the radial vibrations of an ideal sphere. In practice, the filling hole prevents the motion from being radially symmetrical, but the resultant field is too complicated to work with analytically.

More generally, the boundary-layer losses vanish if the pressure is uniform, and in particular, zero, on the interface. For then the tangential component of the pressure-gradient, which is proportional to $\left|\omega u_{t}\right|$, is zero. It would appear then that other things being equal, the boundary-layer losses are smaller the thinner and lighter the envelope, that is, the nearer the fluid boundary is to pressure release.

For the radially symmetrical modes of a cylinder having pressure release ends, the potential is

$$
\psi=J_{0}\left(k_{r} r\right) \cos \frac{n \pi}{2 L} z
$$

in which $n$ is an odd integer. The only nonvanishing component of $u_{t}$ is $\left(u_{z}\right)_{z=a}=-\partial \psi / \partial a$. This is calculated from eq (37) and substituted into eq (36). Integration over the lateral area gives

$$
D_{B}=\frac{n^{2} \pi^{3} a}{2 L}\left(\omega \rho_{L} \mu / 2\right)^{1 / 2} J_{0}^{2}\left(k_{r} a\right) .
$$

As expected, $D_{v}=0$ if the pressure, proportional to $J_{0}\left(k_{r} r\right)$, is zero at $r=a$. We compare this to the loss in the liquid itself, which according to eq (14) is given with sufficient accuracy by

and get

$$
(\lambda+2 \mu) k^{4} \int_{V} \psi^{2} d V
$$

$\frac{D_{B}}{D_{L}}=\frac{3 n^{2} \pi^{2}}{4(2 L)^{2}} \frac{\left(2 \omega \rho_{L} / \mu\right)^{1 / 2}}{k^{4} a} \frac{\alpha_{c 1}}{\alpha} \frac{\left(k_{r} a\right)^{2}\left[1-\left(\omega_{0} / \omega\right)^{2}\right]^{2}}{R^{2}+\left(k_{r} a\right)^{2}\left[1-\left(\omega_{0} / \omega\right)^{2}\right]^{2}}$ 
In obtaining eq (39) we have again made use of the resonance equation, eq (35) and written $\alpha_{c 1} / \alpha$ for $4 \mu / 3(\lambda+2 \mu)$.

Comparison of eq (39) with eq (34) shows that the ratio of the boundary-layer loss to the anelastic loss in the envelope varies as $\omega^{3 / 2}$. Thus we might expect that the loss in the envelope predominates at low, and that in the boundary layer at high, frequencies, and that both are eventually negligible.

Computation shows that in a practical case the mode number may be high enough so that although the loss in the envelope is negligible, the viscous boundary layer loss is still considerable. This viscous loss might then be calculated by the method outlined above and the results corrected accordingly. Mulders [1950] made some interesting measurements on water in a cylinder $28 \mathrm{~cm} \mathrm{O.D.} \mathrm{by} 10 \mathrm{~cm}$ high. The reverberation method (excitation over a band of frequencies) was used at mean frequencies of 750,1010 , and $1500 \mathrm{kHz}$, corresponding to very high-order, densely packed modes. Mulders calculated the viscous boundary loss on an "equipartion" basis for a rigid wall and obtained good results even at the lowest frequency of $750 \mathrm{kHz}$, for which the boundary layer loss was almost half the total.

\section{Discussion}

The resonator-decay method for the measurement of sound absorption in liquids is known from experience to have serious drawbacks. Our analysis, rough though it is, elucidates the mechanism of some of the losses hitherto considered adventitious, and thus provides a rationale for the design of experiments.

We have shown that the basic theory of the method, usually taken for granted, is in fact only approximate for realistic boundary conditions, but that the errors resulting from its application are small for sufficiently high mode numbers. Much more serious are the anelastic losses in the envelope. These are relatively enormous for low-order modes, but become negligible for modes of sufficiently high order. The same is true for losses in the viscous boundary layer. Although we cannot calculate these in the case of a sphere, for which the boundary condition is not uniform owing to the presence of the filling hole, we get a fair idea of the nature of the effect from the analysis of a cylinder. In any case, this loss can be minimized by minimization of the mass of the envelope relative to that of the liquid.

In our example we have chosen water as a test liquid. Water, having relatively low values of $\alpha$ and of $\alpha / \alpha_{c 1}$, gives a "worst-case" type of example, and the unwanted losses are relatively much less in complex liquids having high viscosities and high ratios of compressional to shear viscosity. The envelope material used in the example, Pyrex, having a $Q$ in the range 2000 to 3000 , is typical. A higher $Q$ is of course better, but for a given setup, a large increase in this $Q$ results in a disappointingly small reduction in the lowest frequency at which the losses are acceptable.

The major problems of the method are inherent. The experiments are easier to perform at the lower-order modes because the modes are well separated and easier to identify, and because the demands on the accuracy of the geometry are tolerable, but the lower the mode number, the greater the unwanted losses. Even when the method is used differentially, using a "standard" liquid for which the losses are supposedly known, most workers have had to avoid the lower-order modes.

Another matter of concern is that in the experiment we have used as an example [Wilson, 1951], there is a residual loss of about 40 percent at the high-order modes. It is doubtful that this is due to conduction of sound by the supports and the discrepancy remains unexplained. Moen [1951] in an experiment similar to Wilson's (water, 12-l spherical flask) also found a residual loss of about 40 percent. However, he found greater losses in smaller flasks, and by plotting the decay rate against reciprocal radius and extrapolating the resulting straight line to $1 / a=0$, obtained values of $\alpha / f^{2}$ in agreement with those measured in the tens of megahertz range even at $150 \mathrm{kHz}$, the lowest frequency he worked with. Similar results, namely that the residual loss is proportional to the surface-to-volume "ratio" of the resonator, were obtained by Moen for several other liquids. Thus it appears that this loss is a boundary effect not accounted for by our analysis.

Mulders' [1950] results, described in section 4, are of great interest. He considered that the good accuracy of his calculations of viscous boundary layer loss, based partly on the supposition of a rigid wall, furnished empirical proof that the walls behaved as though rigid, and called this an "amazing fact". According to our analysis (sec. 3.) this behavior is to be expected under the conditions of Mulders' experiment. ${ }^{4}$

The lively interest in possible laboratory measurements of absorption at low frequencies, say down to 1 $\mathrm{kHz}$, has recently intensified. The results of our analysis make us pessimistic about this possibility, especially for low loss liquids, except perhaps by methods as yet undreamt of. Mulders' [1950] experiment, properly scaled to $\mathrm{l} \mathrm{kHz}$, would require a tank having dimensions of tens of meters. To keep such a volume clean, free of air bubbles, and at uniform temperature would tax the resources of even a laboratory which could afford to build the tank.

The $Q$-values of several empty flasks vibrating in the breathing mode were measured by Carl E. Tschiegg. He also made numerous measurements, not extensive enough to report here, on the vibrations of a liquid filled barium-titanate cylinder. These served to verify the general nature of the variation of the losses with mode number as developed in the text.

The work was supported in part by the Office of Naval Research.

\footnotetext{
${ }^{4}$ However, we have considered only axisymmetric modes. An unsymmetrical mode of the liquid, such as occurs in Mulders' experiment, drives a flexural mode of the envelope at a frequency much higher than its natural frequency. Hence its impedance is very high and its motion very low. Of course this is only an informed conjecture.
} 


\section{References}

Lambert, Robert F. (1953), J. Acoust. Soc. Am. 25, 1068-1083

Leonard, R. W. (1946), J. Acoust. Soc. Am. 18, 252.

Leonard, R. W. (1950), Technical Report No. 1, UCLA Physics Department.

Mason, W. P. (1950), Piezoelectric Crystals and their Application to Ultrasonics (Van Nostrand, New York) pp. 393-395, 482.
Moen, C. J. (1951), J. Acoust. Soc. Am. 23, 62-70.

Morse, P. M. and Ingard, K. U. (1968), Theoretical Acoustics (McGrawHill, New York) p. 286

Mulders, C. E. (1948), Appl. Sci. Res. B1, 149-167.

Mulders, C. E. (1950), Appl. Sci. Res. B1, 341-357.

Wilson, Jr., O. B. (1951), Technical Report No. 4, UCLA Physics Department.

(Paper 76C1 \&2-329) 